\title{
A natural experiment of dietary change
}

\author{
Social disruption during the COVID-19 pandemic triggered highly varied changes in diet and lifestyle. It has offered \\ a unique opportunity to study drivers of behaviour change in the general population, building the crucial evidence \\ base needed to tackle major challenges for transforming food systems.
}

\section{Pauline F. D. Scheelbeek}

$\mathrm{R}$ apid transformational changes of the UK food system are needed to improve population health and deliver on critical climate targets. Yes, despite having clear guidelines on what constitutes a healthy ${ }^{1}$ and sustainable $\operatorname{diet}^{2}$, very few people adhere to these recommendations ${ }^{3}$. Furthermore, there is an absence of empirical evidence on the major drivers of transitions from current to healthier, more sustainable dietary patterns in the general population, and no evidence on how these drivers differ across socio-economic groups.

Now, writing in Nature Food, Mazidi and colleagues ${ }^{4}$ advance our understanding of drivers of behaviour change towards healthier (and unhealthier) diets and lifestyles. Using the COVID-19 pandemic as "a unique natural experiment", the authors evaluate self-reported changes in diets, alcohol use, physical activity and sleeping patterns of adults in the United Kingdom and United States, comparing pandemic times with the pre-pandemic situation. The authors framed their interpretation of these outcomes by the level of social disruption the COVID-19 restrictions reportedly caused to the study participants.

Contrary to popular opinion, Mazidi and colleagues found that a substantial proportion of study respondents actually improved their diets and lifestyle behaviours during the COVID-19 restrictions.

Approximately one-third of the respondents reported weight loss during the pandemic, often associated with increased consumption of fruit, vegetables, legumes and wholemeal foods. The 32\% that experienced weight gain often reported more frequent alcohol consumption and increased snacking compared with pre-pandemic times.

An interesting detail is that many who reported dietary improvements specifically mentioned an increase in vegetable (and, to a lesser extent, fruit) consumption as one of the main components of their dietary change. This is important. Higher consumption of fruit and vegetables reduces the risk of several cardiovascular diseases, cancer and all-cause mortality ${ }^{5}$. Vegetables are also pivotal for the transition that lies ahead for the United Kingdom, from diets high in animal-sourced foods to diets that align better with healthy and sustainable dietary guidelines ${ }^{2}$. Furthermore, vegetables, and in particular those that can be grown domestically, would fit well in a food system that is more resilient to the impacts of climate change and other disruptions. This finding can give us hope that, with careful planning and targeted interventions, it may be possible to bring about the substantial population-wide increases in vegetable consumption that population and planetary health requires.

A study from the Food Foundation found that local vegetable shops and vegetable box schemes were responsible for the peri-pandemic increase in vegetable consumption observed in the United Kingdom ${ }^{6}$. But it was noted that uptake was much higher among wealthier households, and socio-economic divides in distribution were evident. Although hypothesized and discussed by many behaviour change scholars (for example, ref. ${ }^{7}$ ), the main reasons and mechanisms for the reported increase in vegetable consumption remain unknown.

Mazidi and colleagues found that the direction and scale of overall pandemic-associated dietary change were independent of deprivation status in their study sample. They found that poor diet before the pandemic was associated with the largest improvements in diet and lifestyle during the pandemic, suggesting that, with the right approach and with the needs of several societal groups in mind, there are possibilities here to enable diet and lifestyle improvements in groups disproportionately affected by diet-related ill health. This would potentially help address and reduce some of the inequalities so typically seen in health across societal groups.

The sizeable differences and directions of dietary behaviour change at the individual level found by Mazidi and colleagues are probably illustrative for the challenges that lie ahead for population-wide shifts towards healthy and sustainable diets. Interventions to target dietary change will be far from a 'one size fits all' matter. And yet, the public interest in healthy and sustainable diets has increased around the world, including in the United Kingdom. The willingness to reduce red and processed meat consumption and achieve higher vegetable intake has increased over the past decade ${ }^{8}$, although there remains a substantial gap between figures on willingness and figures on actual behaviour change?

While the study of Mazidi and colleagues certainly advances our understanding, the key question of why disruption to normal life during the pandemic did trigger a positive dietary behaviour change in one-third of the study's respondents, and worse dietary change in a further third, remains unanswered. Engaging the public to further unravel the underlying mechanisms of noted behaviour change seems an urgent next step to inform policy and practice in the long term. Furthermore, this will improve preparedness for what may happen at the 'end' of the pandemic, when some of the motivations of behaviour change will cease to exist or be reversed.

As Mazidi and colleagues point out, the pandemic has given us an unexpected opportunity to study health behaviour under altered circumstances. The fact that certain groups and individuals, especially those reporting poor pre-pandemic diets, managed to shift to healthier and more sustainable and resilient diets could provide us with crucial evidence going forward. It is extremely important to use this opportunity to expand on the dialogue with the public and 'strike while the iron is hot'. Their experience could be essential to inspire others and accelerate the urgently needed food system transformations for population health, environmental sustainability and food system resilience.

\footnotetext{
Pauline F. D. Scheelbeek (ID) $ه$

Centre on Climate Change \& Planetary Health,
}

London School of Hygiene \& Tropical Medicine, 


\section{London, UK.}

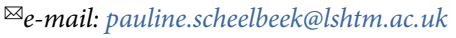

Published online: 16 December 2021

https://doi.org/10.1038/s43016-021-00399-2

References

1. Eatwell Guide (Public Health England, 2016); https://go.nature. com/3oUGVml
2. Willett, W. et al. Lancet 393, 447-492 (2019).

3. Scheelbeek, P. et al. BMJ Open 10, e037554 (2020).

4. Mazidi, M. et al. Nat. Food https://doi.org/10.1038/s43016-02100398-3 (2021).

5. Oyebode, O., Gordon-Dseagu, V., Walker, A. \& Mindell, J. S. J. Epidemiol. Community Health 68 , 856-862 (2014)

6. Wheeler, A. \& Williams, R. Veg Voice Report: Covid Veg (Peas Please and Food Foundation, 2021); https://go.nature. com/3FIbQbi
7. Leone, L. A. et al. Int. J. Environ. Res. Public Health 17, 7397 (2020).

8. Sanchez-Sabate, R. \& Sabaté, J. Int. J. Environ. Res. Public Health 16, 1220 (2019).

9. Yonder Consultants Eating Better Survey: Results (accessed 26 September 2021); https://go.nature.com/3j04olf

Competing interests

The author declares no competing interests. 